\title{
Short Study on Performance of Air Surveillance Augmented by Twin Radars
}

\author{
BALAJTI István ${ }^{1}$
}

\begin{abstract}
Detection of targets at low altitudes with a low Radar Cross Section (RCS) such as drones and stealth could challenge Air Surveillance systems and this situation must be addressed in a cost-effective way. This paper summarizes the newly emerged technical challenges of the Control and Reporting Centre (CRC) and gives solutions on how to overcome recognized shortcomings. In addition it highlights problems and requirements related to drone maneuvering capabilities and their very low RCS. This article summarizes challenges and gives a synthesized solution to the network centric approach based on the short distance Gaussian bi-static radar concept called twin radar coherent signal fusion. The idea is to explore advantages of the twin radar concept such as doubled detection range, increased, superior angular resolution of the system and increased plot update frequency. Examples for the twin radar performance demonstration are based on VHF radar, which has unique capabilities such as exploitation of multipath and resonance frequency for drones. A few peculiarities of the new signal processing are introduced with the extended air traffic control and monitoring network structure.
\end{abstract}

\section{Introduction-motivation}

New challenges for air surveillance of CRC (and Civilian Air Traffic Control - CATC) have emerged in the last decade. The main outcomes of the air target detection, tracking, and recognition problems can be characterized as follows:

- Risk of accidents in the low altitude of long range air surveillance radar networks shall be reduced.

- Natural disaster monitoring and rescue support in case of earthquakes, flood and forest fire.

- Increased interest for control and monitoring drones with less than $20 \mathrm{~cm}^{2}\left(8\right.$ inch $\left.^{2}\right)$ Radar Cross Section.

Populations of the homemade drones or civil and commercial UAVs are rapidly growing. Several features such as flexible route planning, adaptive communications and control mechanism are built in the system. These functions have been designed to allow easy installation and operation keeping the operational cost down. The danger for drone's illegal application such as traditional smuggling, terror threats or more sophisticated airborne cyber-attacks, hijacking mobile phone calls are increasing. Most of the drones are lightweight with very low RCS, due to latest fiber composite technology and the typical size, which is less than $2 \times 4 \mathrm{~m}$ in length and wing span consequently. e.g. in case of the drone shown in Fig. 1 the length is $1.7 \mathrm{~m}$ and the wing span is $3.7 \mathrm{~m}$.

1 balajti.istvan@uni-nke.hu, Hungary, Budapest, National University of Public Services, Military Science Faculty 


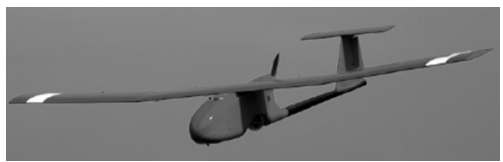

Figure 1. Commercial $U A V^{2}$

Maneuvering capabilities of the drones are increasing beyond man piloted aircraft performance, which redefines the plot update time requirements required for track initiation and maintenance. One of the simplest ways to characterize the required data update time of the highly maneuverable targets is if one determines its plot correlation time, which could be expressed in a simple way as "cos" curve fitting. Details on the target characteristics such as kinematic properties of its maneuvering capabilities and tracking could be found in [1]. Usually the Civilian Air Traffic Centre takes care of the airplanes having acceleration maximum $3 \mathrm{G}$ and are well equipped by IFF/SSR systems. Fig. 2 shows that a target with low speed e. g. $500 \mathrm{~km} / \mathrm{h}$, has no correlation among updated plots at a $2 \mathrm{G}$ maneuver if the radar network plot update is $12 \mathrm{sec}$. The reliability of track initiation and maintenance is critical in case of intensive false plot production even with a well-designed Kalman Filter in the region of NO CORRELATION among updated plots. Consequently, Air Traffic Safety needs an air surveillance radar network augmented by a new type of radar such as twin systems to detect, track and recognize all types of flying objects. [2]

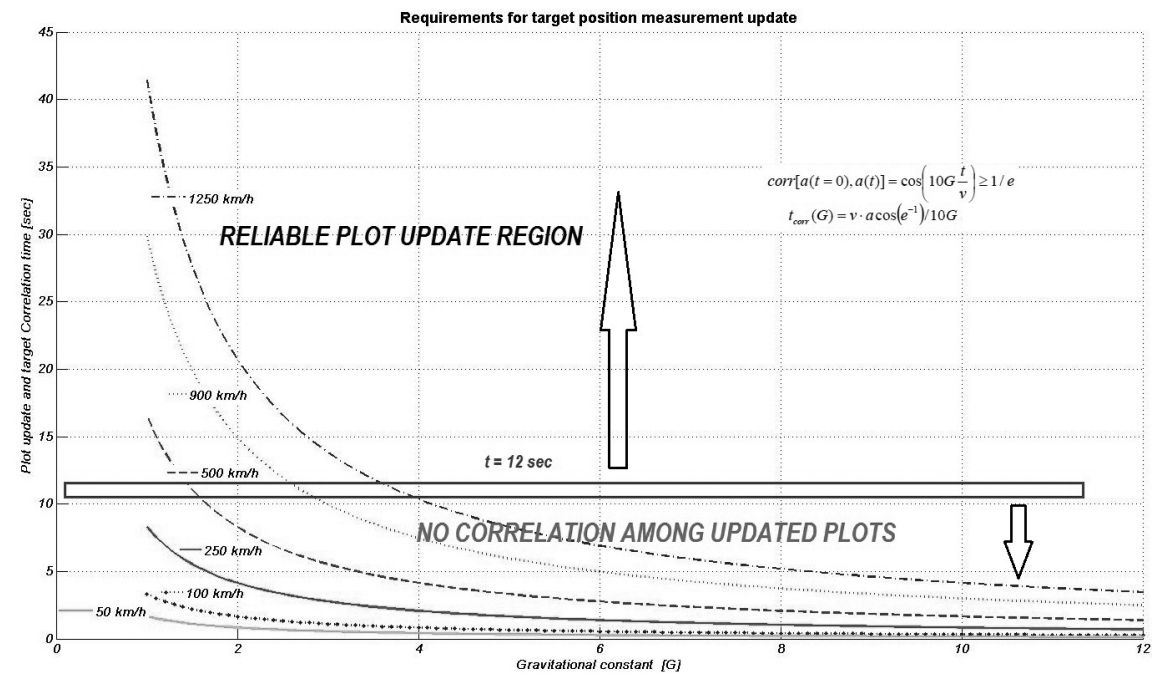

Figure 2. Plot update requirement for reliable tracking [16: 20, updated - upd.] 


\section{Characterization of traditional and twin radar performances}

The traditional way to solve newly emerged detection, tracking and non-cooperative target recognition problems is to increase the required Signal-to Interference-Noise power Ratio (SINR), while the radar concept designer's task is to maximize available technical solutions and advantages at the lowest cost and development risk. The receiver noise is determined as:

$N_{R}=k_{B} T_{S} B_{n}=E\left[w(n)^{*} w(n)\right]=E\left\{|w(n)|^{2}\right\}$

Where:

$k_{B} \quad$ - Boltzmann-constant,

$T_{s} \quad$ - System noise temperature,

$B_{n} \quad$ - The effective noise bandwidth of the receiver,

$w(n)$ - Receiver noise after ADC (Analogue Digital Converter) by “ $n$ ”-sample,

$E\left[w(n)^{*} w(n)\right]=E\left\{|w(n)|^{2}\right\}$ - Auto-covariance of the receiver noise as the complex conjugate representation of the samples.

A simplified situation of the twin radar concept is plotted in Fig. 3. Two identical radars (e. g. Air Traffic Control radar or mobile VHF) are interconnected and are working in coherent signal processing mode for transmit and receive. Antenna beam pointing, surveillance methods and the triggers of transmit and receive signals are synchronized. The two radars have common exciters and signal processors next to the mono radar equipment, with maximum similarity in order to obtain maximum cross-correlation between the dedicated channels.

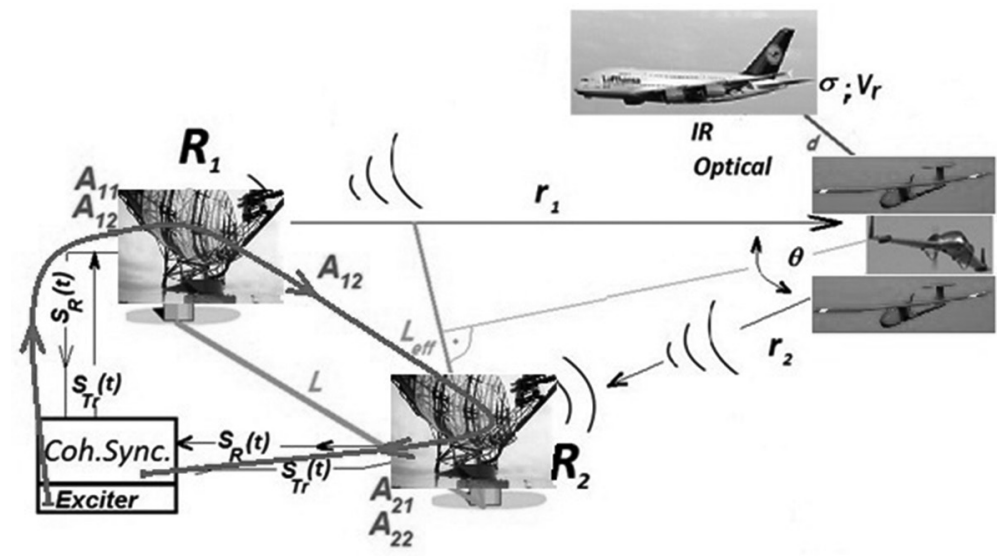

Figure 3.

Short baseline (Gaussian) bi-static topology of twin VHF radar system [16: 73] 
Where:

$\Theta$ - aspect angle $<0.3^{\circ}$, because $L_{e f f}<<r_{1} \cong r_{2}$ This parameter separates the bi-static concept from twin radar concept.

$R_{1}-$ Radar $1, R_{2}-$ Radar 2 ,

$S_{R}(t)$ - signal power received by radar antenna: $S_{R 1}\left[\begin{array}{l}1 \\ \rho\end{array}\right]=S_{R 2}\left[\begin{array}{l}1 \\ \rho\end{array}\right]$;

$S_{T r}(t)$ - signal power transmitted to the antenna: $S_{\operatorname{Tr} 1}\left[\begin{array}{l}1 \\ \rho\end{array}\right]=S_{\operatorname{Tr} 2}\left[\begin{array}{l}1 \\ \rho\end{array}\right]$;

$\rho^{\mathrm{T}}[1 \rho]$ - Correlation coefficient between $R_{1}$ and $R_{2}$,

$\rho\left(t_{1}-t_{2}\right)$-Correlation transfer function of the envelope detector signal between time interval $t_{1}$ and $t_{2}$,

$A_{11} A_{12} A_{21} A_{22}$ - Correlation coefficients in loops: exciter- $R_{1}$-Coh.Sync; exciter- $R_{1}-R_{2}-$ Coh. Sync; exciter- $R_{2}-R_{1}$ - Coh.Sync and exciter- $R_{2}-$ Coh.Sync consequently,

$r_{1}$ - Distance between Radar 1 and Targets,

$r_{2}$ - Distance between Targets and Radar 2,

$L_{\text {eff }}$ - effective base length, the projection length of baseline onto the plane of antennas,

$\sigma$-Radar Cross Section, RCS,

$v_{r}$ - Radial velocity of the target related to radar antennas.

The determining key parameters of the concept are the Coherent Parameter Estimation and the "effective base length" $-L_{\text {eff }}$, which is perpendicular to the bisector of the angle between directions from the target to diverse radars. The modulated signal is generated in the exciter (Coh.Sync./Exciter block), amplified and radiated with the $\mathrm{R}_{1}$ and $\mathrm{R}_{2}$ antennas in the direction of the targets. The electro-magnetic energy is scattered in all directions from the surface of the target, with the energy level determined by the RCS and the Doppler shift proportional to the radial velocity of the target to the directions of separated antennas.

A portion of energy is collected by the $R_{1}$ and $R_{2}$ antennas and the received signal's SINR is increased by amplification, filtering and signal processing gain. After detection, the target's parameter estimation by the system allows target tracking. The realization of this technology is required to manage parameters of the radar equation while keeping the related correlation coefficient high $>0.95$.

\section{Challenges of the concept realization}

It requires centralized control of spatially separated radars with spatially coherent signal processing fully or instantaneously in the case of degraded correlation coefficients. It is necessary for:

- synchronization, phasing and control on mutual coupling/reflection of separated antennas, transmission of reference frequencies and signals that depend on the type of operational mode;

- additional phase shift control of radar subsystems, such as short distance bi-static Doppler and Range peculiarities in ambiguities over relatively long time intervals requires phase adjustment between adjacent systems in order to keep the system correlation coefficient high;

- cooperative beamforming, e.g. VHF multipath condition, signal reception frequency 
and signal waveform emitted by the transmitting radars must be known at the receiving counterpart;

- increased requirements for signal-, data processors, computer systems and data transmission from radars to the common signal processing centre, and for control command distribution;

- coordinate conversion of radar data from local coordinate systems into the common coordinate system, because errors in the determination of antenna positions and the orientation of local coordinate axes influence directly the accuracy of output information traditional phased-array technology which is maximizing the coherent processing gain at the transmitter, beamforming and the receiver side [3].

\section{Symulation results of the realization}

The realization biggest uncertainties are related to the RCS of the target for related correlation coefficients. In narrow-band $\operatorname{radar}\left(\boldsymbol{B}_{n} / \mathbf{f o}^{<}<\mathbf{1}\right.$ at frequency “i”) applications, contributions from all scattering centers combine coherently to produce a single value for the target RCS at every aspect angle. In the proposed multi-band application, a target has target motion and can straddle many range bins, therefore maintaining the coherence of the received signals could be complicated, and may also be limited. Equation (2) depicts that RCS fluctuates as a function of RCS of the $m$ th scattering centre, radar aspect angle on frequency " $i$ " (or in frequency diversity mode of narrow-band radar operation). The individual amplitudes of the electro-magnetic field corresponding to $\sqrt{ } \sigma_{m} i$ at the envelope detector output are added vectorized, taking into account their phases. The complex RCS of the target could be rewritten as $[3,4]$ (Summation by frequency additionally included by author):

$\sigma_{i}(\Theta) \cong\left|\sum_{i} \sum_{m=1}^{M} \sqrt{\sigma_{m i}} \exp \left\{j\left(2 k_{o i} \cos (\theta / 2) d_{m} \vec{r}+\varphi_{m i}\right)\right\}\right|^{2}$

Where:

$\sigma_{m i}-$ RCS of the $m$ th scattering centre for the polarization of the radar on $i$ th narrow-band radar frequency,

$\varphi_{m i}$ - Initial phase shift introduced by $m$ th scattering centre into reflected field on ith narrow-band radar frequency,

“ $m$ " - Refer to scatters $1,2, \ldots$ and subscript " $i$ ” to narrow-band radar operating frequency,

$d_{m}=\left|\left(r_{1}+r_{2}\right)_{m-1}-\left(r_{1}+r_{2}\right)_{m}\right|-$ distances among scattering centers,

$\vec{r}$ - unit vector directed to the radar (does not change in Gaussian bi-static situation,

$k_{o}$ - Wave number, $2 \pi / \lambda_{o}$,

$\lambda_{o}$ - Transmitted wavelength of Radar 1 , or carrier frequency $\left(f_{o}\right), \lambda_{o}=c / f_{o}$

$\exp \left\{j\left(2 k_{o i} \cos (\theta / 2) d_{m} \vec{r}+\varphi_{m i}\right)\right\}=\delta \theta_{m i}$ - Complex representation of the Phase.

Please note: the bi-static RCS of a target, $\sigma_{i}(\Theta)$, at the frequency " $i$ " is equal to its monostatic RCS for a radar located at the bisector of the angle $\Theta$ and working at the wavelength $\lambda_{i} / \cos (\Theta / 2)$, when "the mono-static - bi-static equivalence principle" is valid. 


\section{Findings related to the radar cross section}

The amplitude at the output of the $i-$ th narrow-band radar receiver will be proportional to $\sqrt{\sigma_{m i}}$ and depending also on other parameters of the radar equation marked by $K_{i}$. It is known by basic radar theory that the SINR is the Key Measure of the Radar Performance and defined as ratio of signal power to noise power at the receiver output and the total energy ratio for coherent signals can be calculated. Fig. 4 shows the genetic receive and signal detection structure of the twin radar. The system has up to $X_{I}(t)$ simultaneous channels and $M F-$ Matched Filters, where $X_{1}(t)$ and related transmit part is the reference. Compared to reference the time delays and the phase shifts are measured, adjusted to improve the signal coherency in transmit, beamforming and receive for maximal SINR. The envelope detector " $\Sigma$ " sum the signals in phase and comparison with the threshold detects the targets in the traditional way.

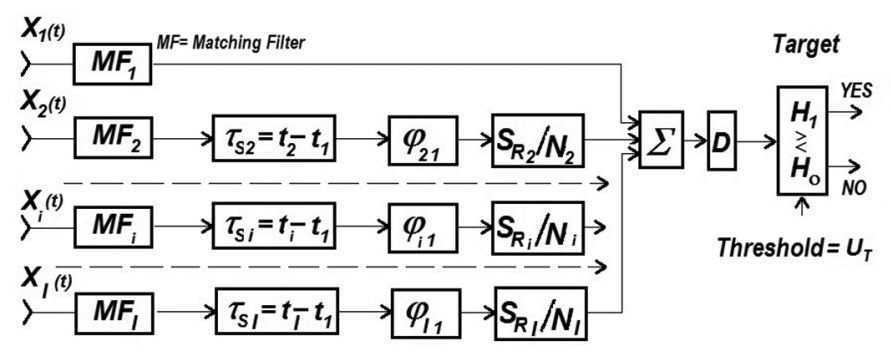

Figure 4.

Receive and signal detection structure of the twin radar [3: 123, upd.]

In case of coherent signal processing an envelope-detector receiver output amplitude from the narrow-band such as "VHF", "L" and "S"band radar receivers are added. It would be possible to define a composite RCS as:

$$
\begin{aligned}
& \sigma_{i}(\Theta) \cong \frac{1}{\sum_{i} k_{B} T_{S i} B_{n i}}\left|\sum_{i} \sum_{m=1}^{M} \sqrt{K_{i} \sigma_{m i}} \delta \theta_{m i}\right|^{2}=\ldots \\
& \ldots=\frac{1}{\sum_{i} k_{B} T_{S i} B_{n i}}\left|\sum_{m=1}^{M} \sqrt{K_{V H F} \sigma_{m V H F}} \delta \theta_{m V H F}+\sqrt{K_{L} \sigma_{m L}} \delta \theta_{m L}+\sqrt{K_{S} \sigma_{m S}} \delta \theta_{m S}\right|^{2}
\end{aligned}
$$

Where the term $\sum k_{B} T_{S i} B_{n i}$ represents the summed noise powers of the three (“ $i$ " - in general case) narrow-band radar receiver outputs. Let's assume that the two sets of radar parameters are equivalent with $K / k_{B} T_{s} B_{n}$. In case of noncoherent signal integration a squaredetector receiver output amplitude from the radar receivers is added. The total RCS can be expressed as:

$$
\begin{aligned}
& \sigma_{i} \cong \sum_{i}\left(\frac{\sqrt{K_{i}}}{k_{B} T_{S i} B_{n i}} \sum_{m=1}^{M}\left|\sqrt{\sigma_{m i}} \delta \theta_{m i}\right|^{2}\right)=\ldots \\
& =\left|\frac{K_{V H F} \sigma_{V H F}}{k_{B} T_{S V H F} B_{n V H F}} \delta \theta_{V H F}\right|^{2}+\left|\frac{K_{L} \sigma_{L}}{k_{B} T_{S L} B_{n L}} \delta \theta_{L}\right|^{2}+\left|\frac{K_{S} \sigma_{S}}{k_{B} T_{S S} B_{n S}} \delta \theta_{S}\right|^{2} \mid
\end{aligned}
$$


Putting the total RCS value into the radar equation the system output signal amplitude can be normalized to "r. m. s" noise amplitude. Equations (3) and (4) show that the temporal correlation is determined by the target rotation angle during time on target interval and by the target dimensions to the wavelength ratio.

A first-order approximation of RCS for a complex object can be obtained by summing the major specular RCS components. This is shown in Fig. 5 with a simulation performed on spheres in the VHF frequency band, showing RCS changes relative to the angle of radar pulses. The 3 small spheres are 9.84 inches $(25 \mathrm{~cm})$ and the two big spheres are 13.8 inches (35 cm) with the positions and parameters of Fig. 5 .

The random aspect of the target RCS is the result of a rotation from the mean position of the target through a small random angle. Averaging should be made over the random target aspects, which are small in our cases, relative to spatially diverse antennas. The spatial correlation function of signal fluctuations for 3D moving targets of an arbitrary shape proves that a target RCS containing many scatterers is not random.

The target's RCS dependency on the frequency band, shows advantages if the target size determined by the resonance region of the allocated frequency. The key performance is that the RCS signal fluctuation is relatively steady for a large aspect angle relative to the radar. Consequently random and fluctuating signals at the receiver input are a result of summing many partial signals from small non-random scatterers with sharply changing phases.

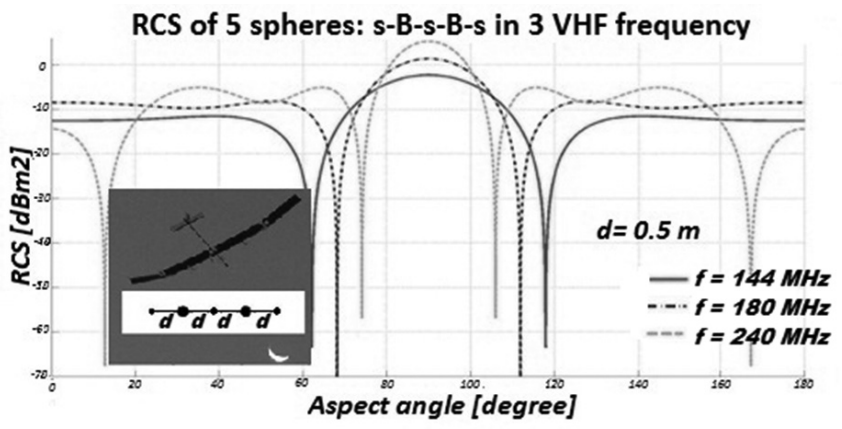

Figure 5. Drone RCS models based on 5 spheres in 3 VHF frequency (no source $-n$ s.)

Further investigation is required with the comparison of advantages and disadvantages to find the proper frequency for the required radar system for dedicated targets recognition. Fig. 6 shows plots of the composite RCS of the modeled spheres. Table 1. summarizes findings of the analyzed scatterers signal processing statistics related to situation displaced at Fig. 6 . 


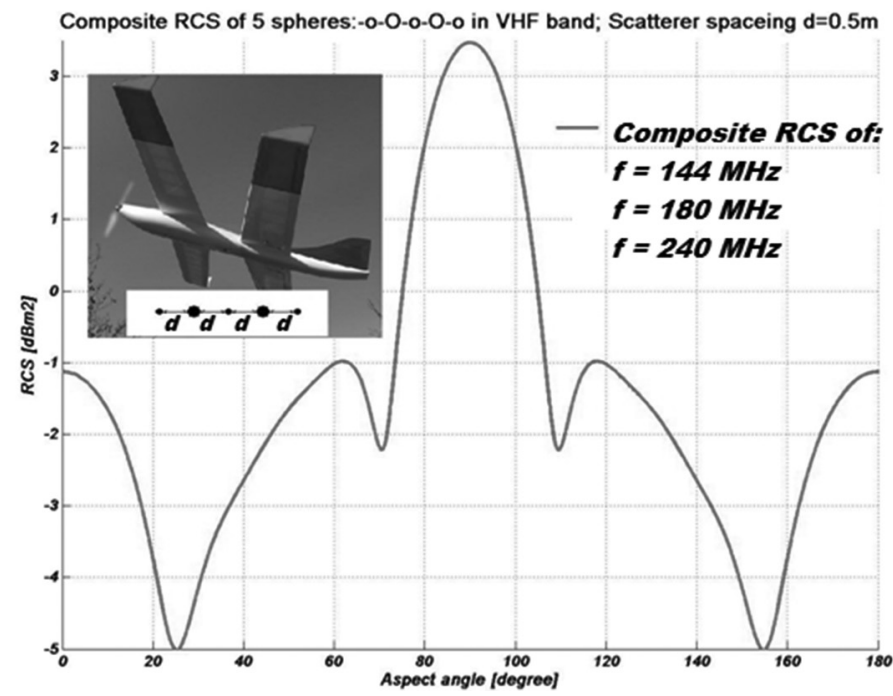

Figure 6. Composite RCS statistics for twin VHF radar signal processing (n s.)

\begin{tabular}{|c|c|c|c|c|}
\hline RCS statistics: & $\mathbf{f = 1 4 4} \mathbf{~ M H z}$ & $\mathbf{f = 1 8 0} \mathbf{~ M H z}$ & $\mathbf{f =} \mathbf{2 4 0} \mathbf{~ M H z}$ & “Composite” \\
\hline MEAN & -12.36 & -8.899 & -9.425 & -1.513 \\
\hline median & -12.19 & -8.819 & -7.376 & -1.637 \\
\hline mode & -28.54 & -11.85 & -67.67 & -5.02 \\
\hline STD & 6.483 & 5.75 & 8.695 & 2.099 \\
\hline
\end{tabular}

Table 1. Twin VHF radar signal processing statistics

The main advantages of the twin VHF radar composite signal processing are the increased RCS and the moderated signal fluctuation, which increases the achievable SINR of twin radars.

Further information on the bi-static and multi-static radar could be obtained from [5, 6]. Note: applicability of the concept on the VHF radar has some peculiarities "well known" by radar experts that require attention and detailed clarification such as the multipath effect, mutual coupling, reflections and environmental interferences that frequently block the new VHF radar developments.

\section{IV./b. Findings related to the twin antenna patterns}

Fig. 7 shows two conventional phased-array radar which are connected to twin radar phased arrays. The whole transmitting array is considered as one array (Resultant Directivity Pattern), which has an overall illumination function containing two identical illumination functions related to $R_{1}, R_{2}$ and a few hundred " $m$ " isolated gaps between. The receive patterns contain sum and simultaneous different channels. Frequency diversity modes are foreseen with 3 waveforms emitted at $\pm 3 \mathrm{~dB}$ beamwidth shift to the center beam. The uplink and downlink channels are to be separated by traditional beamformer networks. The two radars, 
introduced above are interconnected and working in coherent signal processing mode as simplified situation is plotted in Fig. 3. The phased array antenna patterns have been analyzed in the literature so far [6] and do not needed deep explanation. Consider a phased array with $M$ transmit and receive elements, where the beamformer produces its output by forming a weighted combination of signals:

$y(n)=\sum_{m=1}^{M} c_{m}^{*} x_{m}(n)=c^{H} x(n) \rightarrow$

In case of twin phased array, such as Fig.7:

$2 \sum_{m=1}^{M} c_{m}^{*} x_{m}(n)+D^{*}(n)$ - the antenna gain is doubled compared to a mono-radar case because the beam forming networks of the joint phased array complex conjugate pattern productions are common.

Where: $c=\left[\begin{array}{lllll}\mathrm{c}_{1}^{*} & \mathrm{c}_{2}^{*} & \mathrm{C}_{3}^{*} & \ldots & \mathrm{c}_{\mathrm{M}}^{*}\end{array}\right]^{T}$ - column vector of beamforming weights, $x_{m}(n)-$ signal on the phased array element $m$ and $D^{*}(n)$ - is the complex mutual coupling/reflection coefficient of two phased arrays.

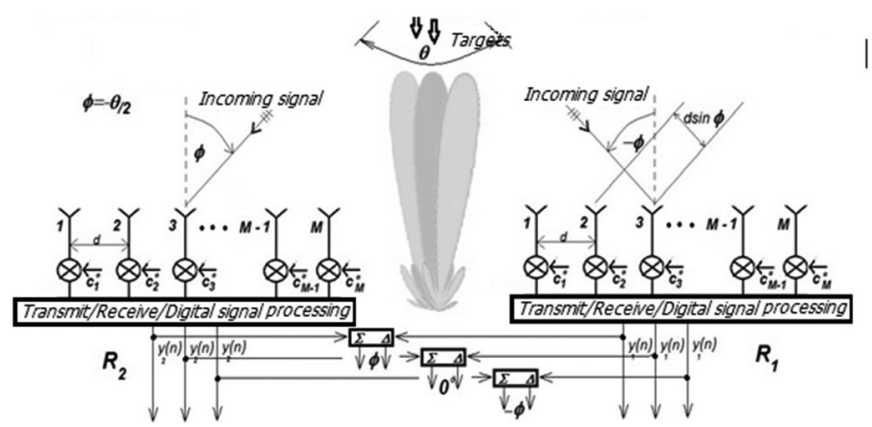

Figure 7. Phased array of twin radar (unpublished - up.)

To demonstrate the peculiarities a simplified simulation was carried out where the phased array of $R_{1}$ and $R_{2}$ radar contain 32 elements with the same illumination functions of $R_{1}$ and $\mathrm{R}_{2}$. The distance between the phased array antennas is $500 \mathrm{~m}$, where the real part of the complex mutual coupling/reflection coefficient is $0.3,0.1$ and 0.05 respectively. The beams are calculated using Fast Fourier Transform (FFT). The calculated far field antenna pattern of the two closely located VHF radar antennas is displayed at Fig 8. The receive pattern situation has been simulated and the results analyzed also. The receive SUM channel is identical to the transmit array tapering, while the DEL channel`s array tapering phase of R1 radar which has been shifted by 180 degrees produces the complementary antenna far field pattern.

As predicted by phased array antenna theory the "grating lobes" of the far field patterns are increased. Details of the effect can be seen in Fig. 9. The main beam pattern has deep notches with a magnitude of 20-30 dB which can be observed. These notches cause additional signal fluctuations regarding the complementary channel delta, in case of operational frequency change. The effect of mutual coupling/reflection between two phased arrays is changing by antenna positions, which has significant effect on the antenna patterns as Fig. 8 shows. Consequently, the notches in the twin VHF radar main beam one shall be precisely calculated and measured during the calibration of the system and adjusted continuously during operation. 


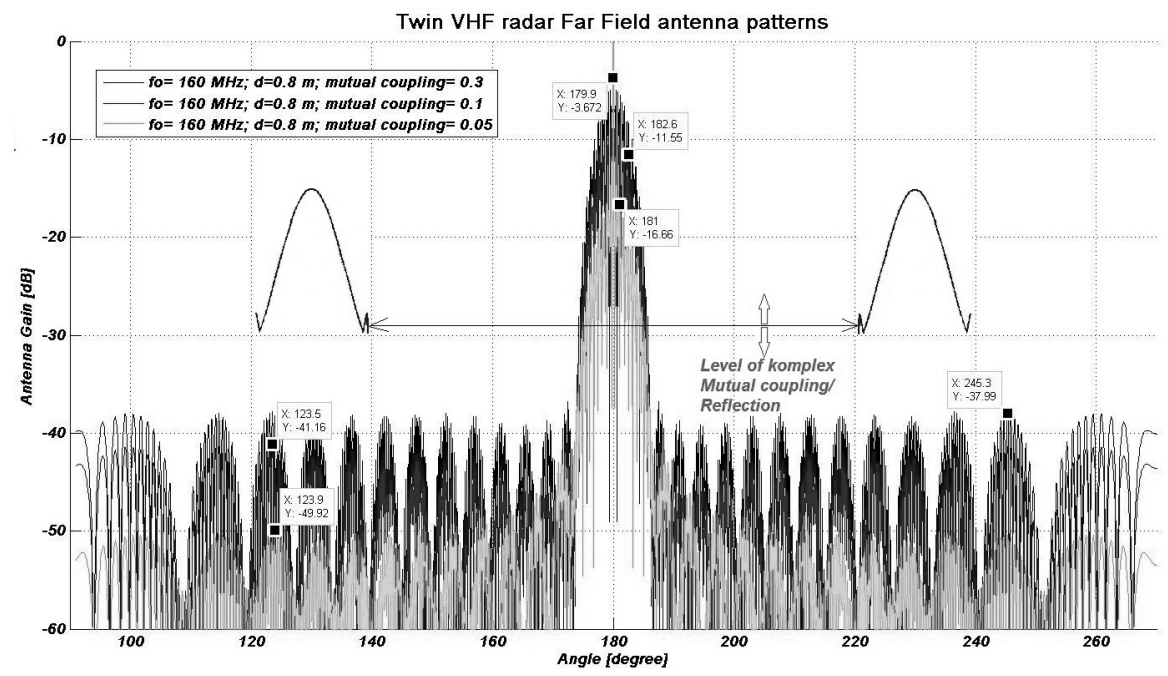

Figure 8. Twin VHF radar far field diagrams with the transmit illumination functions (up.)

The notches in the main beam comparison caused by frequency differences and/or antenna rotation show a similarity to the monopulse angular measurement techniques, where simultaneous offset beams are compared. This effect has to be exploited for improvement of angular accuracy as we see in the case of mono-pulse or multi-beam systems. (In the case of different carrier frequencies the notch positions move with the frequency change giving possibilities for further increase of the angular resolution measurement accuracy of the twin radar system.) See Fig. 9.

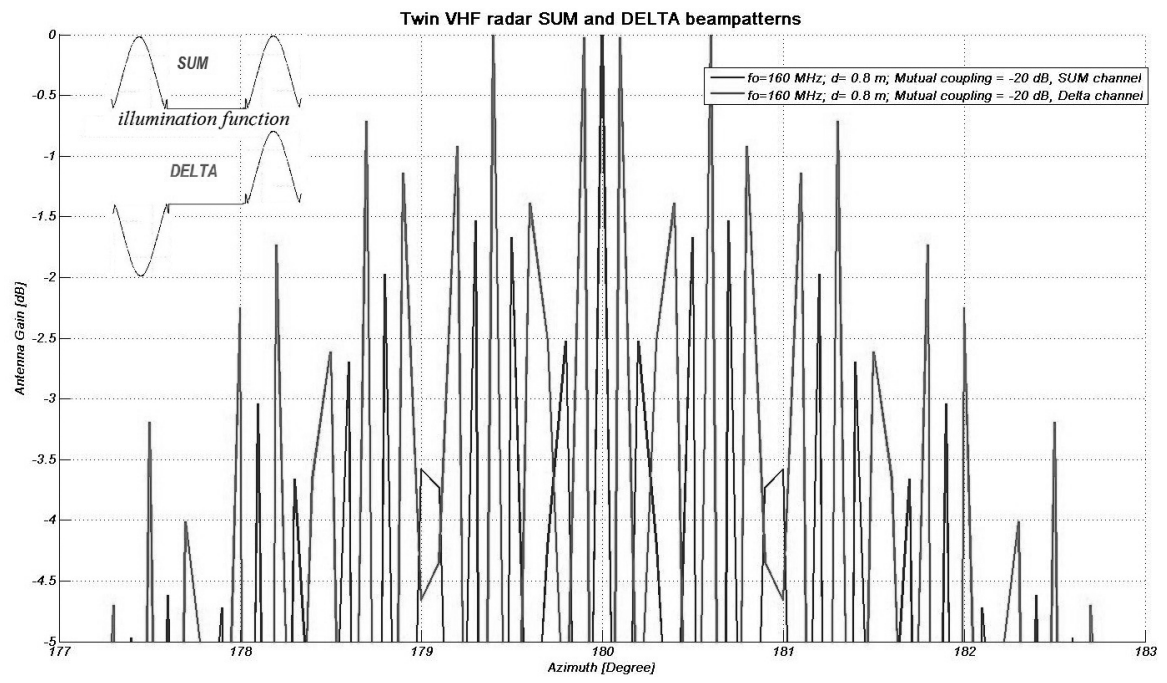

Figure 9. Magnified view of the twin VHF radar far field patterns (up.) 


\section{IV./c. Findings related to Range and Doppler Ambiguities'}

Fig. 10 shows geometry and kinematics of the target motion in a twin radar system as characterized in Fig. 3. As target position relative to the receive site is required to be known in twin radar implementation the Gaussian bi-static Range $\left(R_{u}\right)$ and Doppler $\left(v_{u}\right)$ ambiguity peculiarities at the receivers can be calculated. Both of them depend on the Pulse Repetition Frequency (PRF) of the twin radar system. The ambiguous range in each PRF is followed by an unfolding and correlation process. [7] The unfolding creates a vector of possible ranges for valid detections by adding a set of integers $[0 \ldots K]$ times the unambiguous range interval:

$R_{u}=R_{\text {amb }}+\frac{c}{2 f_{v}}[0 \ldots K]$

$v_{u}=\frac{f_{r} \lambda}{2} \cos (\delta) \cos (\Theta / 2)[-J \ldots 0 \ldots K]$

Where:

$c$ - speed of light, $f_{r}=P R F$, The set of integers $[0 \ldots K]$ are referred to as the range ambiguity number, with $K$ determined by the maximum range of interest, $\lambda$ - operational wavelength of the radar, $f_{r} \lambda / 2$ - is the first blind speed (PRF velocity), $\delta, \Theta$ - related to the target movement in Fig. 10, and [-J......K] represents the set of Doppler ambiguity numbers covering the maximum negative and positive Doppler-velocities for the targets of interest.

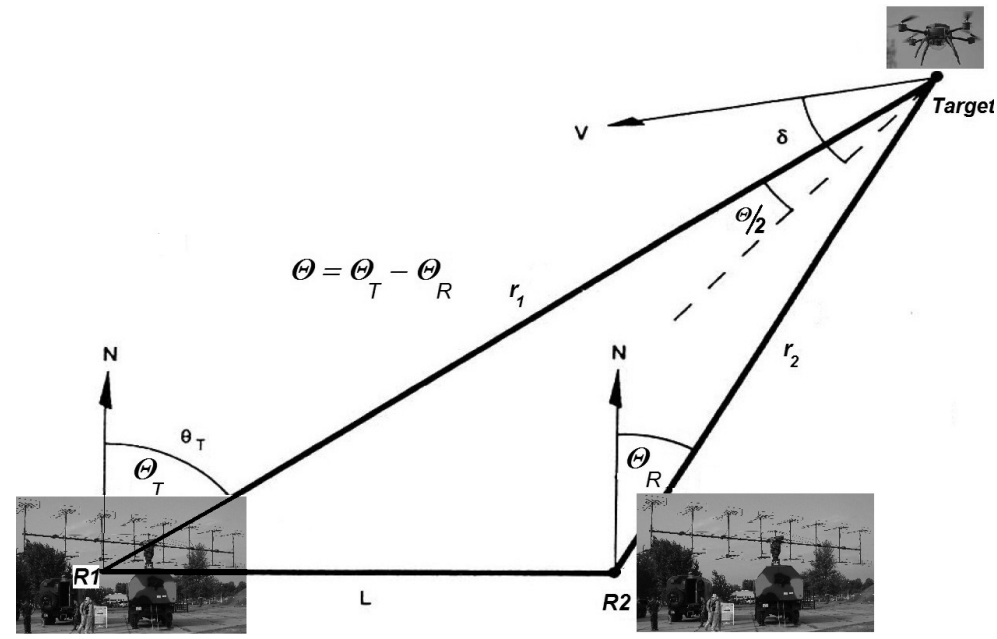

Figure 10. Twin VHF radar and target movement geometry (up.)

As the example assumes: the VHF radar antenna rotation is 6 APM (Antenna rotations Per Minute, operational frequency $=160 \mathrm{MHz}$, Target radial speed $=0.1-1000 \mathrm{~m} / \mathrm{s}$, its amplitude $=100 \mathrm{mV}$ and the target radial speed fluctuate due to change of heading and/or acceleration. Fig. 11 draws the attention to the proper Pulse Repetition Frequency selection complexity while remaining unambiguous of Range and Doppler velocity in the required margin. First of all, the Doppler unambiguous margin is critical up to $100 \mathrm{~Hz}$ PRF for low speed targets and reduces in higher PRF. 
Secondly, the range unambiguous margin has opposite behavior, but both cases are very sensitive for targets at low speed with high acceleration capabilities as speed vector change indicates. (Such targets can be drones and other types of small airplanes that could be problematic from tracking points of view.) This situation could be resolved by increased resolution of the twin radar system.

Increase of resolution performance of the twin radar is convenient to calculate with approximate expression (Resultant Directivity Pattern) based on Rayleigh criterion to echo from point target. [3] The thermal noise error of monopulse estimate, for high SINR can be expressed [8]:

$\sigma_{\alpha} \cong \frac{\Theta_{3}}{k_{m} \sqrt{2(1+S / N) n}}$

Where the

$\sigma_{(\alpha)}$ - denotes the r.m.s. error of the resolution estimation in the bi-static plane,

$\Theta_{3}-$ Resultant Directivity Pattern beamwidth at $3 \mathrm{~dB}$;

$k_{m}$ - denotes the r.m.s. error of the monopulse slope can be determined directly from measured $\Sigma$ and $\Delta$ patterns,

$n$ - denotes the number of the received pulses.

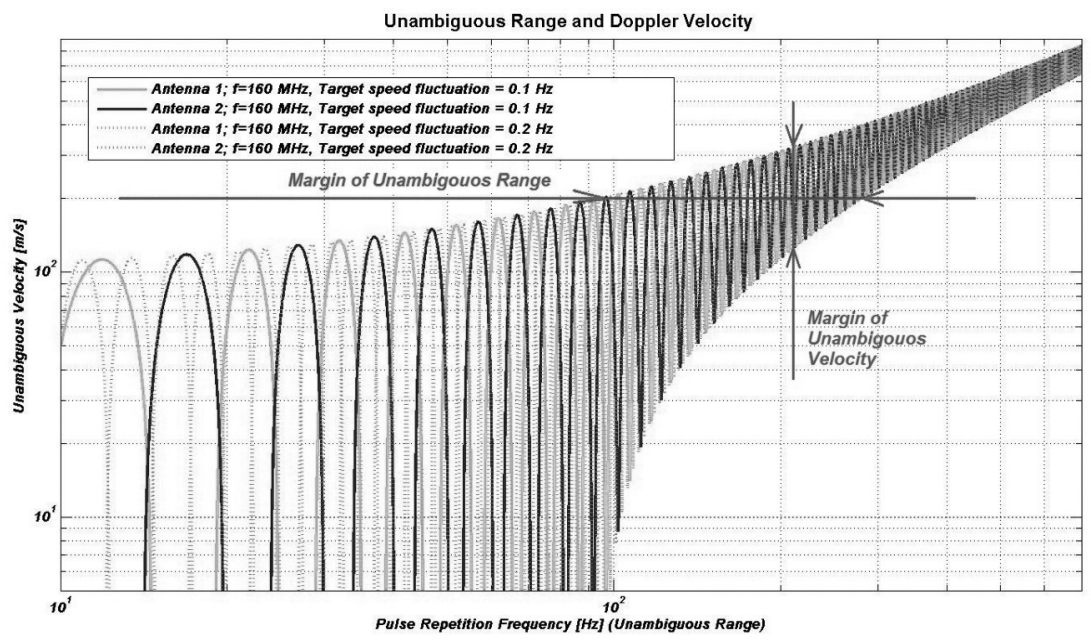

Figure 11. Twin VHF radar unambiguous Range and Doppler velocity vs. Pulse Repetition Frequency (up.)

Detection capabilities of a twin system could be proven by Barton's MRSAS 3.3.2 Program calculations. [9] This method allows easy comparison of the radar system performance changes, while it looks "not important" enough to be implemented for comprehensive studies. The author's experience of this article shows that the "apples and oranges" type of comparison's confusion can be reduced significantly if researchers more frequently use the "old fashioned Blake charts" for showing the Row Order of Magnitude of the applicability of the idea. Related to the subject twin radar performance calculations have been introduced in [10]. 
The summary of the calculations show that the detection range of the target is doubled for default circumstances. The reason is that the new system itself shall have advanced capabilities such as $6 \mathrm{~dB}$ Power-Aperture search advantage in simplified operational mode and $9 \mathrm{~dB}$ Power-Aperture-Gain. Additionally 7-10 dB increase of RCS for same types of targets and 2-2.7 dB multipath effect can be explored in the case of VHF radar band offered circumstances [11].

Additional $3 \mathrm{~dB}$ advantages in the twin radar signal processing can be exploited as pointed out in Fig. 12. This Fig. has been produced applying findings [12] to the MATLAB Phased Array Toolbox.

The $\mathrm{P}_{\mathrm{d}}$ increases from $0.1 \mathrm{~dB}$ to $0.5 \mathrm{~dB}$ at fixed $\mathrm{P}_{\mathrm{fa}}=4 \cdot 10^{-6}$ in twin radar signal processing or the $\mathrm{P}_{\mathrm{fa}}$ reduces from $8 \cdot 10^{-4}$ to $4 \cdot 10^{-6}$ at fixed $\mathrm{P}_{\mathrm{d}}=0.5 \mathrm{~dB}$. A compromise has to be found among the reduced probability of detection, probability of false alarm and the required SINR for a good quality of target detection. One of our future tasks is to characterize the centralized signal fusion with a correlation gate feedback control function applied to three different $\mathrm{Pd}$ (0.9; $0.3 ; 0.1)$ related to "optimal” Pfa of the radars as is it looks to be one of the most promising solutions for twin radar applications.

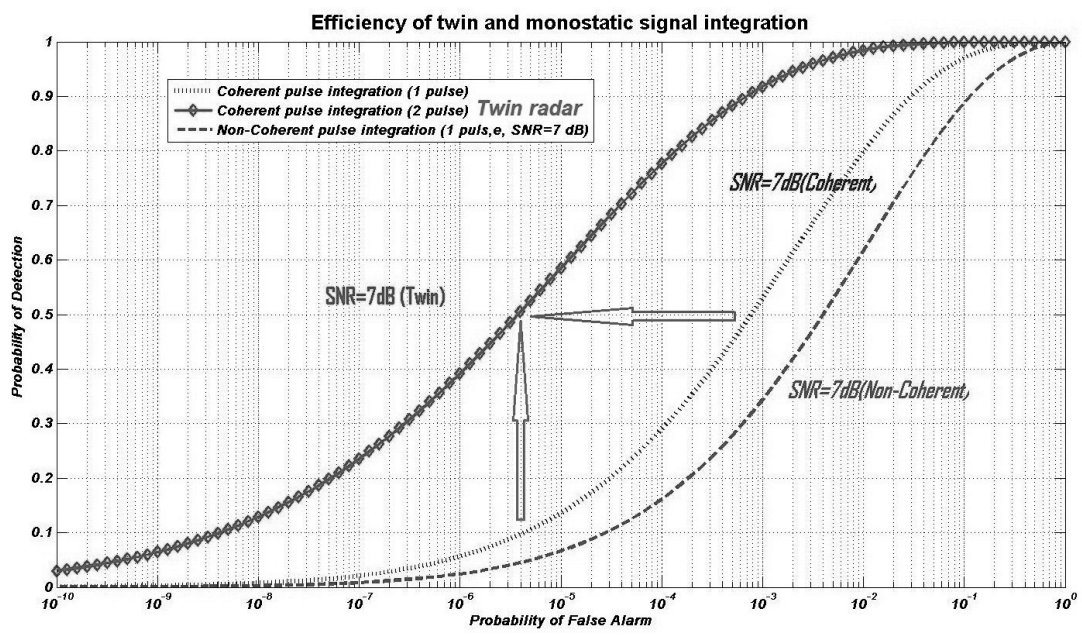

Figure12. $P_{d}$ vs. $P_{f a}$ in case of mono and twin VHF radar cases [16: 80]

Fig.13 depicts the generic structure of the extended military-civilian air traffic control and monitoring network. Different types of narrow-band radar such as short, long range, "VHF", "L" and "S" band radars are connected. The bone radar network contains military and civilian radars that are installed under RADOME and all of them connect to the air traffic control centers for centralized plot fusion. There is no plot exchange among radars in the traditional structure at all. The plot based radar networks performances and capabilities have been analyzed in the literature so far and do not require further explanation.

The extended detection range requires increased performance of the radar which could be enriched by added mobile or fixed counterparts for realization of twin radar offered performance, where the twin passive systems are an essential part of the structure. The method based on twin radar concept offers a sort of coherent video integration in the Sensor Fusion 
Post (SFP) at the signal fusion level, which could be allocated inside, or close to the radars. The twin radar concept advance performances, increase of target detection and angular resolution, could be extended further as is shown in the middle of the figure. The signal level data exchange among radars such as "VHF", "S" and passive ("P") systems are required because hits of the radars are collected from all radar correlation gates, in three different threshold levels, commutatively. This information is then compared, merged, filtered and the targets are extracted with standard target detection requirements. Advance passive radars can be installed on board of drones too. This solution offers extended air surveillance performances from the air for "S", "L" and "VHF" radars applying so called sparse phased array technology on the board of mutually cooperating drones.

The low frequency band such as HF and VHF radars importance are increasing recently in modern air surveillance systems because their RF pulse propagation peculiarities can be exploited for target detection even in extreme weather conditions. Hungary has large flat surfaces that are very suitable for exploitation of the multipath effect of the VHF band radars. Consequently they are very cheap from operational and maintenance points of view and VHF radar type shall be kept in the radar fleet and should be modernized time to time. Nowadays the twin radar concept solves the biggest shortcoming of low frequency band radar because their angular resolution can be increased as equation (8) points out.

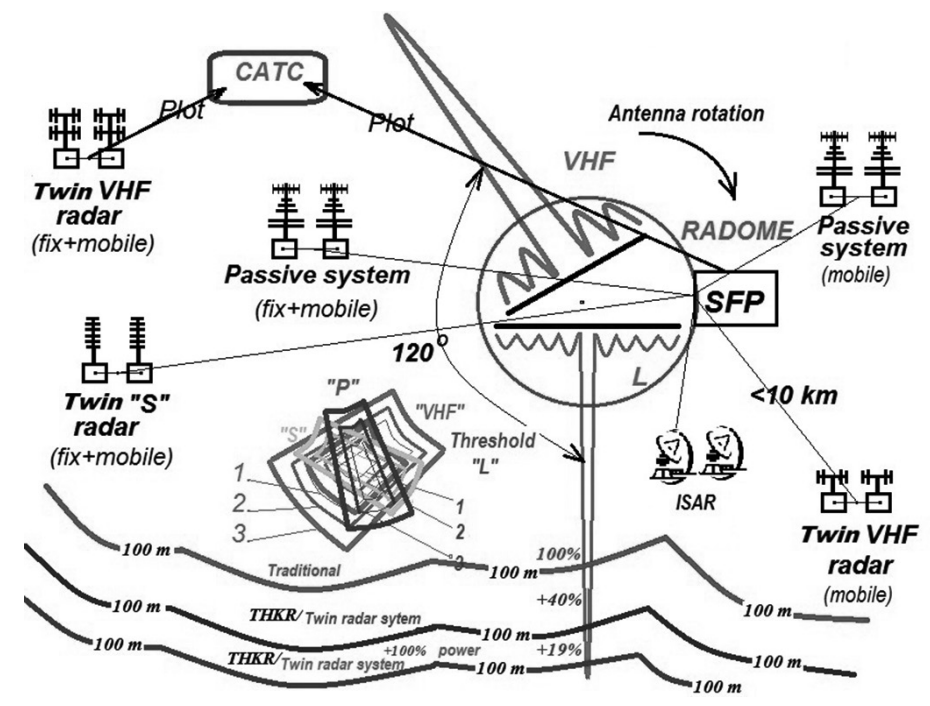

Figure 13. Extended air traffic control and monitoring network structure [16: 84]

This system concept supports the track initiation and maintenance of the highly maneuverable targets too. It solves uncertainties introduced in Fig. 2 because the radars marked under RADOME are synchronized in antenna rotation in such a way that the " $\mathrm{"} \mathrm{band} \mathrm{radar}$ follows the "VHF" band radar surveillance sector with a 120 degree delay. If both radars independent plot update time is $10 \mathrm{sec}$, the target position and speed vector update sequence is 0 sec -3 sec -10 sec -17 sec -20 sec etc. This feature increases the target initialization probability up to $12 \mathrm{G}$. 
Two, Inverse Synthetic Aperture Radar (ISAR) were an integral part of the Hungarian Air Defense system in the past. The ISAR is a known technique to generate a two-dimensional high resolution image of a target. The image of ISAR radar has had sufficient quality to allow it to be used for target recognition purposes. In situations where other radars display only a single unidentifiable bright moving pixel or scope of the target, the ISAR image is often adequate to discriminate between various drones, missiles, military and civilian aircraft. The difficulty in utilizing this capability is the object motion and its periodicity, which is measured in 2-dimensional Fourier transform of the received signal as a function of frequency and target aspect angle. The twin concept could be extended to the Inverse Synthetic Aperture Radar when two ISAR coherently connected and formulate an Interferometric Inverse Synthetic Aperture Radar with 3-dimensional FFT applications. The twin ISAR shown in the Fig. 13 should be an essential part of drone recognition and increased safety measure support of the airplanes in the near future.

The twin radar can also be subject to intentional interference initiated by hostile transmissions. Thus it is essential to examine how the performance will be affected under such an environment. A few aspects of the investigation and related simulations have been already carried out in open literature. [13]

\section{Summary}

General overview on the air surveillance system augmented by twin radar is introduced. The system elements connections and main performances highlight the fact that management of the extended volume of the air surveillance space is required in detection range of the low RCS signature targets, such as drones, Stealth with high maneuvering capabilities and in the very low altitude down to the highway level at low cost. It was pointed out by this article also that the twin radars, which are based on very high correlation coefficients of the transmitter, antenna and receiver sub-systems have unique performances. Most important among these are: the capability of the double detection range of the Stealth, drones constructed with low or very low RCS technologies.

Main challenges of the twin radar concept realization are reviewed and analyzed. Behaviors of the target RCS in case of coherent and non-coherent signal integration is demonstrated, the antenna pattern peculiarities, the Range and Doppler ambiguities are analyzed, and the angular resolution increase is introduced briefly. The results show that newly proposed system itself shall have advanced capabilities such as $9 \mathrm{~dB}$ Power-Aperture-Gain sensitivity advantage and more than $3 \mathrm{~dB}$ signal processing gain. Additionally 7-10 dB increase of RCS for some types of targets and 2-2.7 dB multipath effect can be explored in the case of VHF radar band offered circumstances. The author's field experiences show that the target signatures RCS in the VHF band usually give 5-10 dB stronger reflections than in the "S" band. The twin radar signal processing offers a significant increase in probability of detection, and false alarm reduction. The main drawback of the VHF radar was in the past the angle measurement accuracy and resolution capability which could be eliminated too.

Additional benefits can be obtained at a relatively low-cost, under the assumption that the system is set up by integrating operational radar, or by adding remote Passive Location Capabilities applying the findings of advanced MIMO technologies to the twin radar concept. From a logistic support point of view the performance of the radar systems shall be foreseen 
guaranteed and checked regularly for 20 years. Cost efficient realization of it requires well developed and maintained "in situ" performance measurement technologies, which are required for tests above the Built In TEst (BITE) capabilities of the radar. It is important to note that the twin radar concept gives improved reliability and operational availability of the air surveillance radar systems.

These benefits are among others focused on the possibility to implement fully coherent transmit, antenna, receive and signal processing methods into the wide area distributed radar systems. The topic has got more attention and importance recently as patent [14] and article [15] highlights this fact.

\section{References}

[1] ŐSZ S.: Bevezetés és rendszerparaméter analízis. Budapest: ZMNE, 1998. (in Hungarian)

[2] BALAJTI I., KENDE Gy., SINNER E.: Increased importance of VHF radars in Ground Based Air Defense. IEEE Aerospace and Electronic SYSTEMS Magazine. 271 (2012) 4-18. ISSN 0885-8985 DOI: https://doi.org/10.1109/MAES.2012.6145436

[3] CHERNYAK, V.: Fundamentals of Multisite Radar systems. Philadelphia: Gordon \& Breach Science Publisher, 1998. ISBN 10: 9056991655 DOI: https:// doi.org/10.1201/9780203755228

[4] KNOTT, E. F., SHAEFFER, J. F., TULEY, M. T.: Radar cross section. 2nd Ed. Raleigh: SciTech Publishing, 2004. DOI: https://doi.org/10.1049/SBRA026E

[5] WILLIS, N. J., GRIFFITHS, H. D.: Advances in bistatic radar. Raleigh: SciTech Publishing, 2007. ISBN 1891121480

[6] JOHNSON, R. C.: Antenna Engineering Handbook, 3rd Ed. New York: McGraw-Hill, 1994. ISSN 1063-665X

[7] SKOLNIK, M. (ed.): Radar Handbook, 3rd ed. New York: McGraw-Hill, 2008. Chapter 6

[8] Pod red. BONDARENKO, B.F.: Osznovi Posztroenija RLSZ. Kiev: KVIRTU PVO, 1987.(in Russian)

[9] BARTON, D. K.: Modern Radar System Analysis ver 3.0 Software and User`s Manual, Norwood: Artech House, 2007. ISBN13: 978-1-59693-264-7

[10] BALAJTI I. : Performance of Civilian Air Traffic Control augmented by twin radars. IEEE Radar Symposium (IRS), 19-21 June 2013 549-560. ISBN 978-1-4673-4821-8

[11] KUSCHEL, H.: VHF/UHF radar. 1. Characteristics. Electronics \& Communication Engineering Journal, 142 (2012), 61-72. ISSN 0954-0695

[12] SHNIDMAN, D.A.: Update on radar detection probabilities and their calculation. IEEE Transactions on Aerospace and Electronic Systems, 441 (2008), 380-383. DOI: https:// doi.org/10.1109/TAES.2008.4517013

[13] SELLER R.: Módszerek céltárgyparaméterek rádiólokációs mérési pontosságának növelésére. Budapest: BME, 1996. (in Hungarian)

[14] THOME, G. D., ENYMANN R. P., STEUDEL, F.: System and Method For Coherently Combining Plurality of Radars. US Patent No. US 7,358,892 B2. 15 April 2008.

[15] BROOKNER, E.: MIMO Radar: Demystified. Microwave Journal, Aerospace and Defense Channel, 15 January 2013. http://www.microwavejournal.com/articles/18894-mimo-radardemystified (downloaded 1902 2013)

[16] BALAJTI I. : Napjaink radartechnikája. (Modern radar technologies and twin radars.) http:// hhk.old.uni-nke.hu/index.php/hu/osszes-hir/88-fooldal/795-napjaink-radartechnikaja (downloaded: 1911 2013) 